\title{
Was haben wir in 100 Jahren erreicht?
}

\section{Ein Rückblick des Vereins medical women switzerland (mws)}

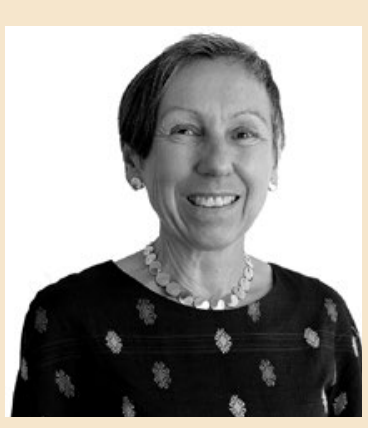

Marianna Bodenmann-Zanetti Vorstandsmitglied mws

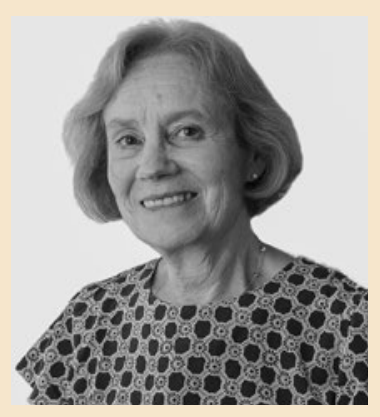

Adelheid Schneider-Gilg Präsidentin Vorstand mws
Die ersten Schweizer Ärztinnen kämpften auch nach dem Staatsexamen um ihre berufliche Anerkennung in der Gesellschaft und besonders bei ihren männlichen Kollegen: Diese verweigerten ihren Kolleginnen die Teilnahme an Weiterbildungsveranstaltungen und fachlichen Diskussionsrunden mit dem Hinweis auf ihr Geschlecht - eine reine Machtdemonstration. Die Ärztinnen liessen sich nicht einschüchtern, sondern organisierten sich umgehend selbst. 1922 entstand daraus die Vereinigung Schweizer Ärztinnen (heute: medical women switzerland, mws).

Der Anteil der Ärztinnen stieg seit dem Studienabschluss von Marie Heim-Vögtlin (erste Schweizer Ärztin) im Jahr 1874 trotz der widrigen Umstände langsam an und betrug in den 1950er Jahren ca. 12\%. Die meisten Ärztinnen, mit und ohne Familie, arbeiteten nach dem Staatsexamen in der eigenen Praxis, wo sie ihr Arbeitspensum und die Kultur am Arbeitsplatz selbst bestimmen konnten. Teilzeitstellen, insbesondere im Rahmen der Weiterbildung, und das Thema der Vereinbarkeit von Beruf und Familie waren tabu. Die seltenen Karrierefrauen, so zum Beispiel die Gründerinnen des Spitals Pflegerinnenschule in Zürich, stachen als grosse Ausnahmen heraus.

In den 1970er Jahren wurde der Druck der Basis, v.a. der Assistenzärztinnen, immer grösser, die Weiterbildung zumindest teilweise im Rahmen von Teilzeitstellen absolvieren zu können. Auch mws-Mitglieder sowie deren Vorstand engagierten sich auf verschiedenen Ebenen für das Anliegen und publizierten über die Schwierigkeiten, die Facharztausbildung zusammen mit der Kinderbetreuung unter einen Hut zu bringen. 1982 wurde die Weiterbildungsordnung (WBO) erfreulicherweise angepasst: Die Hälfte der reglementarischen Dauer der Weiterbildung konnte im Rahmen von Teilzeitstellen von mindestens $50 \%$ absolviert werden. Die einzelnen Fachgesellschaften stellten in den folgenden
Jahren ihre Weiterbildungsprogramme entsprechend um.

Ursula Ackermann, Professorin für Sozial- und Präventivmedizin in Basel, setzte sich als Präsidentin der Schweizer Ärztinnen in den 1980er Jahren unermüdlich für die Umsetzung der neuen WBO und die Schaffung von Teilzeitstellen in Spitälern ein. Sie war begeistert, als 1984 in der SÄZ zwei Ärzte (!) über ihre Erfahrungen als Job-SharingPartner berichteten. Es war der Beweis, dass solche Modelle auch in der Schweiz umsetzbar sind.

Tatsache ist, dass seit 2005 mehr Frauen als Männer das Medizinstudium abschliessen (2019: 58\% Frauen und 42\% Männer). Heute wollen nicht nur Frauen, sondern auch Männer ihre Weiterbildung teilweise im Rahmen von Teilzeitstellen absolvieren. 2015 waren es 25\% der Assistenzärzte und $42 \%$ der Assistenzärztinnen. Tatsache ist aber weiterhin, dass das Angebot an Teilzeitstellen vielerorts bis heute ungenügend ist. Als Gründe oft genannt werden Kontinuitätsprobleme und administrative Unsicherheiten - ob das die wahren Gründe sind, sei an dieser Stelle dahingestellt.

Die neue WBO 2020 ermöglicht, die ganze Weiterbildung in Teilzeit, bis zu zwölf Monaten sogar in Pensen von $20 \%$ bis $49 \%$, zu absolvieren. Damit ist nach Jahrzehnten des Kampfes um Weiterbildung in Teilzeit das Ziel theoretisch erreicht. Es fehlt aber leider nach wie vor an der konkreten Umsetzung, das heisst an Teilzeitstellen in allen Fachgebieten.

Nebst der Weiterbildung beschäftigen uns Ärztinnen noch weitere spezifische Probleme, zum Beispiel und ganz besonders die sogenannte "gläserne Decke»: Warum gibt es immer noch so wenige Frauen in den höchsten Kaderstellen, in wissenschaftlichen Stellen mit universitärer Laufbahn, in der Standespolitik?

Diese Problematik ist nicht Schweiz-spezifisch, sondern in der ganzen westlichen Welt aktuell. Und, ehrlich gesagt, liegt es nicht nur an der männlichen Hierarchie (die wir natürlich hinterfragen und anzweifeln), sondern auch an uns Frauen selber: Wir müssen es vermehrt wagen, Verantwortung zu übernehmen und unsere Ziele energisch zu verfolgen!

Als Fazit lässt sich festhalten, dass wir zwar schon viel erreicht haben, aber noch lange nicht an unseren Zielen angelangt sind. Wir müssen weiterkämpfen, für unsere Rechte und auch für unsere Pflichten. 